\title{
Strategic Network Formation through an Intermediary*
}

\author{
Elliot Anshelevich and Onkar Bhardwaj and Koushik Kar \\ Rensselaer Polytechnic Institute \\ 110 8th Street, Troy NY 12180 \\ eanshel@cs.rpi.edu,bhardo@rpi.edu, koushik@ecse.rpi.edu
}

\begin{abstract}
Settings in which independent self-interested agents form connections with each other are extremely common, and are usually modeled using network formation games. We study a natural extension of network formation games in which the nodes cannot form connections themselves, but instead must do it through an intermediary, and must pay the intermediary to form these connections. The price charged by the intermediary is assumed to be determined by its operating costs, which in turn depend on the total amount of connections it facilitates. We investigate the existence and worstcase efficiency (price of anarchy) of stable solutions in these settings, and especially when the intermediary uses common pricing schemes like proportional pricing or marginal cost pricing. For both these pricing schemes we prove existence of stable solutions and completely characterize their structure, as well as generalize these results to a large class of pricing schemes. Our main results are on bounding the price of anarchy in such settings: we show that while marginal cost pricing leads to an upper bound of only 2, i.e., stable solutions are always close to optimal, proportional pricing also performs reasonably well as long as the operating costs of the intermediary are not too convex.
\end{abstract}

\section{Introduction}

Settings in which independent self-interested agents form connections with each other are extremely common, and range from computer networks to social networks to economic networks. Such settings are usually studied using network formation games: a rich category of games which studies properties of networks resulting from agents (nodes) forming relationships (edges) in a strategic manner to maximize their utility (or minimize their cost). The extensive body of work on network formation games (see [Jackson, 2005] for a survey) looks at many different settings, different notions of node utility (e.g. [Jackson and Wolinsky, 1996;

${ }^{*}$ The work was partially supported by NSF awards CCF1101495 and CNS-1218374.
Fabrikant et al., 2003]), different concepts of network stability (e.g., [Epstein et al., 2007; Calvó-Armengol and İlkılıç, 2009]), convergence dynamics (e.g., [Dutta et al., 2005; Derks et al., 2008]), etc. For example, the classic work of [Jackson and Wolinsky, 1996] considers an abstract setting in which both agents must agree to form a connection or link between them, but a single agent can break this connection; this work was later extended in many works such as [Bala and Goyal, 2000; Watts, 2003] and forms the foundation of the network formation game which we consider in this work.

The above work on network formation games assumes that agents can form links (connections, relationships, etc.) themselves. The key question that we study in our work, however, is what happens when the agents cannot form connections themselves, and must instead do it by paying an intermediary. This occurs, for example, when traders exchanging goods need to pay fees to the freight companies, and in many other settings (see below) when agents are paying some centralized service for their connections, instead of forming connections themselves. If the intermediary charges exorbitant fees which exceed the benefits of forming connections, then nodes would rather not establish connections at all. On the contrary, the work mentioned above can be interpreted as the intermediary being absent, or connections having fixed costs. We are interested in the natural case when the fees charged by the intermediary are determined by its operating costs.

A major motivation for our work comes from Internet Service Providers (ISPs) forming connections via Internet Exchange Points (IXPs). In most basic terms, IXPs are data centers with huge network switches through which ISPs form connections with each other in order to exchange traffic. In return, IXPs recover their operating costs (which depend on the size of infrastructure needed) by charging fees to each member ISP. The recurring component of these fees typically depends on the capacity of the port(s) allocated to each ISP, see e.g., [LINX, 2015; DEC-IX, 2015]. This interaction between ISPs and IXPs is extremely important for the modern Internet: a considerable portion of Internet traffic [Ager et al., 2012; Cardona Restrepo and Stanojevic, 2012] passes through an IXP, and thus requires two ISPs to form a connection with each other by paying an IXP to forward their traffic. In essence an IXP can be thought of as a marketplace in which the seller (the IXP) sells capacity for forming connections to its clients (the ISPs): this is exactly the setting of 
network formation through an intermediary that we study in this work (see Related Work for more details).

Model Summary. We now outline the model for our network formation game with an intermediary. We assume that the cost of each agent (i.e., node) consists of two components: the connection cost incurred due to all the connections it forms with its neighbors, and the payment made to the intermediary. For any pair of nodes we assume that as the strength of their connection increases, the connection cost incurred by each endpoint due to this connection decreases (e.g., as a pair of ISPs exchange traffic at a higher rate, the losses due to endcustomer dissatisfaction decrease; as people form a stronger relationship, they get more benefit from this relationship, etc). Our concept of a stable solution in this setting is a natural extension of the notion of pairwise stability from [Jackson and Wolinsky, 1996]: Using $y_{i j}$ to denote the connection strength between a pair of nodes $(i j)$, we say that a solution is stable if no pair of nodes $(i j)$ is able to increase the strength $y_{i j}$ of their connection in a mutually beneficial way (i.e., to lower the cost for both of them), and no node benefits by reducing the strength of any of its connections. Note that how changing the strength of a connection affects the cost of a node depends not only on the change in the connection cost, but also on the change in the payment made to the intermediary.

We are particularly interested in the case where the payments made by each node to the intermediary are determined by the operating costs of the intermediary. Motivated partly by ISP-IXP interactions, where the operating cost of an IXP depends on the size of the infrastructure needed to carry the traffic of all ISPs, we assume the operating cost of an intermediary to be a function $c(y)$, where $y$ is the total strength of connections formed by all the nodes. Given this, we will focus on the following two popular notions of pricing:

- Proportional pricing: Let $y_{i}$ denote the total strength of the connections formed by a node $i$. In proportional pricing, the operating cost $c(y)$ of the intermediary is split among the nodes such that the payment made by a node $i$ to the intermediary is proportional to $y_{i}$, i.e., node $i$ pays $\frac{y_{i}}{y} c(y)$.

- Marginal cost pricing: In marginal cost pricing, node $i$ makes a payment of $c^{\prime}(y) \cdot y_{i}$ to the intermediary.

We will also briefly discuss Equal pricing where each node makes a fixed payment $c(y) / n$ to the intermediary (where $n$ is the number of nodes), regardless of connection strengths.

Our goal in this work is to investigate the existence and quality of stable solutions in which payments to the intermediary conform to the above pricing schemes. Towards measuring the quality of a solution, we define the social cost of a solution as the sum of costs of all the nodes and the intermediary (the cost of the intermediary is its operating cost minus the total payment it receives). Thus the optimal solution for a given instance is the solution which minimizes the social cost (note that since payments cancel out, the optimal solution does not depend on the pricing schemes). We use the popular notion of Price of Anarchy (PoA) to measure the worst-case efficiency of a pricing scheme: the PoA of a pricing scheme is the maximum possible ratio of the social cost of a stable solution (with this pricing scheme) to the social cost of the optimal solution.

Our Contributions. We define network formation games with an intermediary, which are a natural generalization of the basic model in [Jackson and Wolinsky, 1996] as discussed in Related Work. We investigate the existence and PoA of stable solutions in which payments made to the intermediary satisfy well-known notions of proportional pricing and marginal cost pricing. We show that despite requiring the payments to satisfy these pricing schemes, stable solutions always exist for both pricing schemes. In fact, we show a stronger result that stable solutions exist for any pricing scheme in which the payments made by any node $u$ are of the form $p(y) \cdot y_{u}$, where $p(y)$ is any continuous and increasing function. We also provide a characterization of all stable solutions for any such pricing scheme, in which we show that any stable solution can be generated recursively from other stable solutions. Our main results are on bounding the worst-case efficiency (PoA) of the above pricing schemes. We show that under marginal cost pricing PoA is upper bounded by 2, and thus all stable networks of connections are close to optimum in cost. We also show that under proportional pricing PoA is upper bounded by $\max \left(2, \sup _{y \geq 0} \frac{y c^{\prime}(y)}{c(y)}\right)$. For example, under a common assumption that the operating cost $c(y)$ of the intermediary is a convex polynomial of degree at most $d$, this bound evaluates to at most $d$, and thus proportional pricing becomes very reasonable as long as the operating costs of the intermediary are not too convex.

\section{Related Work}

Network formation games have been extensively studied in the literature under many different settings, see [Jackson, 2005; Page and Resende, 2013; Tardos and Wexler, 2007] and the references therein.

Our work is most heavily influenced by the classic network formation game introduced in [Jackson and Wolinsky, 1996]. This work considers a setting in which two nodes can form links with mutual consent (if it benefits both of them) but any node can sever the connection if it hurts its utility. Our network formation game with intermediary (including the notion of stability) can be viewed as a natural extension of this abstract setting in the presence of an intermediary. Note that we also allow connections with fractional strengths instead of just integral strengths $\{0,1\}$, which is more natural for both networks of Internet traffic and social networks. Whereas [Jackson and Wolinsky, 1996] investigate the structure of the stable solutions and the conditions for which the stable solutions are also optimal, we give bounds on inefficiency of stable solutions with intermediary prices satisfying the well-known notions of proportional and marginal cost pricing. The notions of pairwise stability and price of anarchy have also been used in many works related to network formation games such as [Fabrikant et al., 2003; Calvó-Armengol and İlkılıç, 2009; Corbo and Parkes, 2005; Anshelevich and Hoefer, 2012].

A major motivation for our work is how internet service providers (ISPs) form connections through internet exchange points (IXPs). A typical IXP architecture corresponds to an 
ethernet switch through which member ISPs can exchange traffic [Augustin et al., 2009; Ager et al., 2012]. In return, each member ISP makes a payment to the IXP of which the recurring charges are determined by the port capacities requested by ISPs: see the following sample fee structures [LINX, 2015; AMS-IX, 2015]. Then each member ISP can form connections with a subset of other ISPs in order to exchange traffic [Ager et al., 2012]. A large number of IXPs, especially in Europe, are operated on a non-profit basis [Ryan and Gerson, 2012] and the operating costs of an IXP are largely determined by the cost of the infrastructure needed for traffic exchange [EuroIX, 2015].

Our setting is generally part of the enormous body of work on markets and pricing (see e.g., [Johari, 2007; Vazirani, 2007] and the reference therein). Unlike this work, however, we consider a seller who is selling pairwise connections instead of items to individual buyers, and thus must analyze pairwise equilibrium solutions with all of the complexities that this entails.

\section{Model and Preliminaries}

Let there be $n$ nodes. Denote by $y_{i j}$ the strength of the connection between nodes $i$ and $j$. Let $y_{i}=\sum_{(i j) \ni i} y_{i j}$ denote the total strength of connections formed by node $i$ and let $y=\sum_{i} y_{i}$ denote the total strength of connections formed through the intermediary. In our primary motivation of the ISP-IXP example, $y_{i j}$ is analogous to the traffic between two ISPs and $y_{i}$ is analogous to the total traffic exchanged by an ISP. The cost of each node is assumed to be the sum of the following two components:

- Connection cost: As discussed above, we assume that for any pair $(i j)$ of nodes as $y_{i j}$ increases, the connection cost incurred by each endpoint due to this connection decreases. We model the connection cost incurred due to connection $(i j)$ as $\lambda_{i j}\left(B_{i j}-y_{i j}\right)$, which is a linear function of the connection strength $y_{i j}$. This connection cost is incurred by both end points. Here $B_{i j}$ is the budget or maximum allowed connection strength for the pair $(i j)$. For the ISP-IXP setting, $\lambda_{i j} \cdot B_{i j}$ is analogous to the benefit obtained by each ISP after exchanging a total traffic $B_{i j}$ committed to the end-users. Then $\lambda_{i j}\left(B_{i j}-y_{i j}\right)$ is analogous to the losses incurred by each ISP for failing to deliver the committed amount of traffic.

- Payment to the intermediary: We will focus on proportional pricing and marginal cost pricing as discussed in the Introduction. Both these pricing schemes can be expressed as $\gamma \cdot y_{i}$ where $\gamma=c(y) / y$ for proportional pricing and $\gamma=c^{\prime}(y)$ for marginal pricing (recall that $c(y)$ denotes the operating cost of the intermediary for a total connection strength of $y$ ). This can be also interpreted as each node paying a price $\gamma$ per unit connection strength. This thinking is consistent with the observation that typically an IXP charges an ISP based on the capacity of allocated port(s), and thus the charges are proportional to $y_{i}$. We will call $\gamma$ as the price of the intermediary.

Let $\vec{y}$ denote the vector of connection strengths $y_{u v}$ of all the pairs, which we will also refer to as allocation vector. We will call a tuple $(\vec{y}, \gamma)$ of an allocation vector $\vec{y}$ along with an intermediary price $\gamma$ as a solution. Based on the above discussion, the cost of a node $i$ in a solution $(\vec{y}, \gamma)$ is given by

$$
C_{i}(\vec{y}, \gamma)=\gamma \cdot \sum_{(i j) \ni i} y_{i j}+\sum_{(i j) \ni i} \lambda_{i j} \cdot\left(B_{i j}-y_{i j}\right)
$$

We assume the operating cost $c(y)$ of the intermediary to be a strictly convex function of total connection strength $y=$ $\sum_{i \neq j} y_{i j}$, with $c^{\prime}(0)=c(0)=0$. Taking into account the total payments from the nodes, the cost of the intermediary is then given by

$$
X(\vec{y}, \gamma)=c(y)-\gamma \cdot \sum_{i} \sum_{(i j) \ni i} y_{i j}
$$

We define the social cost of an allocation vector $\vec{y}$ as the sum of the costs of the nodes and the intermediary. Note that since the payments cancel out, this summation is a function of only $\vec{y}$. Thus the social cost of an allocation vector $\vec{y}$ is given by

$$
S C(\vec{y})=c(y)+\sum_{i} \sum_{(i j) \ni i} \lambda_{i j} \cdot\left(B_{i j}-y_{i j}\right)
$$

Drawing a parallel to the capacity of the physical link connecting ISP $i$ to the IXP, we will assume a capacity of $L_{i}$ for each node, i.e., that $y_{i} \leq L_{i}$ for a node $i$. In more general networks, the capacity $L_{i}$ corresponds to the total amount of connections that an agent can possibly make and maintain. Thus an instance of the network formation game with an intermediary is completely specified by $(\vec{\lambda}, \vec{B}, \vec{L}, c)$ where $\vec{\lambda}$ is the vector of variables $\lambda_{i j}, \vec{B}$ is the vector of budget variables $B_{i j}, \vec{L}$ is the vector of node capacity constraints $L_{i}$ and $c(y)$ is the cost incurred by the intermediary for maintaining a total connection strength of $y$.

We now describe the notion of a stable solution. In the network formation game analyzed by Jackson and Wolinsky [Jackson and Wolinsky, 1996], a stable network was defined as a network where no pair of nodes could add an edge to lower the cost of both, and no node could remove an adjoining edge to lower its own cost. Analogous to this definition (as well as similar definitions of stability in other works [Corbo and Parkes, 2005; Calvó-Armengol and İlkılıç, 2009]), we consider a solution to be stable in our setting when no $(i j)$ pair of nodes can increase $y_{i j}$ to lower the cost (of both of them), and no node can reduce $y_{i j}$ to lower its own cost. We formally define below the notion of a stable solution. We use $\tau_{\text {min }}^{i}(\vec{y})$ to denote $\min \left\{\lambda_{i j}:(i j) \ni i\right.$ and $\left.y_{i j}>0\right\}$, i.e., the minimum $\lambda_{i j}$ among the pairs adjoining node $i$ having a connection with positive strength.

Definition 3.1 A solution $(\vec{y}, \gamma)$ formed by the allocation vector $\vec{y}$ and intermediary price $\gamma$ is said to be a stable solution whenever every pair of nodes $(i j)$ satisfies the following conditions:

1. $y_{i j}>0$ implies $\lambda_{i j} \geq \gamma$

2. $y_{i j}<B_{i j}$ implies either $\lambda_{i j} \leq \gamma$ OR at least one of the endpoints, say $i$, satisfies $y_{i}=L_{i}$ and $\lambda_{i j} \leq \tau_{\min }^{i}(\vec{y})$.

To understand above definition, let us first consider the simpler scenario when all $L_{i}$ are infinite. In this scenario, there 
is no limit on how much total connection strength any node is allowed to have, thus the only limiting constraint for a pair of nodes is the intermediary price and their own budget $B_{i j}$. Consider the following equation obtained from Equation 1:

$$
\frac{\partial C_{i}(\vec{y}, \gamma)}{\partial y_{i j}}=\gamma-\lambda_{i j}
$$

Thus the relation between $\gamma$ and $\lambda_{i j}$ dictates whether increasing or decreasing the connection strength $y_{i j}$ would benefit a pair by lowering the cost of both endpoints: if $\lambda_{i j}<\gamma$ then a pair would benefit by decreasing $y_{i j}$ whereas if $\lambda_{i j}>\gamma$ then a pair would benefit by increasing $y_{i j}$ (See Equation 4). Thus in a stable solution $y_{i j}>0$ implies $\lambda_{i j} \geq \gamma$ because if $\lambda_{i j}<\gamma$ then both the endpoints would decrease $y_{i j}$ to lower their cost. Similarly, we obtain that $y_{i j}<B_{i j}$ implies $\lambda_{i j} \leq \gamma$ for the simplified scenario of all $L_{i}$ being infinite.

Introducing finite node capacity constraints $L_{i}$ does not change the first condition for stability (i.e., $y_{i j}>0$ implies $\left.\lambda_{i j} \geq \gamma\right)$. However with finite capacity constraints, even when increasing $y_{i j}$ can benefit a pair (i.e., $\lambda_{i j}>\gamma$ ), one of the endpoints, say $i$, may not agree on doing so if it is using all its capacity for more beneficial connections (i.e., $y_{i}=L_{i}$ and $\left.\lambda_{i j} \leq \tau_{\text {min }}^{i}(\vec{y})\right)$. This implies that if $y_{i j}<B_{i j}$ then either $\lambda_{i j} \leq \gamma$ (same as the case with no capacity constraints) or one of the endpoints, say $i$, satisfies $y_{i}=L_{i}$ and $\lambda_{i j} \leq \tau_{\text {min }}^{i}(\vec{y})$.

To measure the quality of stable solutions, we will use the popular measure of Price of Anarchy (PoA) (see for example [Papadimitriou, 2001; Koutsoupias and Papadimitriou, 1999]). Let $\overrightarrow{y^{*}}$ be the allocation vector which minimizes the social cost (see Equation 3) given an instance $I$. Then the price of anarchy for marginal cost pricing is given by

$$
\operatorname{PoA}=\sup _{I} \sup _{\substack{(\vec{y}, \gamma) \text { is stable } \\ \text { s.t. } \gamma=c^{\prime}(y)}} \frac{S C(\vec{y})}{S C\left(\overrightarrow{y^{*}}\right)}
$$

In other words, the PoA (for marginal cost pricing) is the maximum factor by which the social cost of a stable solution (with marginal cost pricing) can differ from the social cost of an optimal solution. We can define PoA similarly for other pricing schemes. Now we proceed to characterizing the structure of stable solutions, and later bounding their PoA.

\section{Characterization and Existence of Stable Solutions}

In this section, we investigate the existence and provide a characterization of stable solutions in which payments made to the intermediary satisfy well-known notions of proportional pricing and marginal cost pricing. We show that despite requiring the payments to satisfy these pricing schemes, stable solutions always exist for both pricing schemes. In fact, we show a stronger result in Theorem 4.3: that stable solutions exist for any pricing scheme in which the payments made by any node $i$ are of the form $p(y) \cdot y_{i}$, where $p(y)$ is any continuous and increasing function. In Theorem 4.1, we provide a characterization of all stable solutions, which shows that any stable solution can be generated recursively from other stable solutions.
Let us introduce some notation to begin with. We will use $\lambda^{k}$ to denote $k^{t h}$ biggest value from the set of $\lambda_{i j}$ variables for a given instance. We will also use $\lambda^{0}$ to denote any arbitrary value greater than $\lambda^{1}$. For example, if there are three pairs in an instance with $\lambda_{i j}$ values $\{16,16,15\}$ then $\lambda^{0}$ is any value greater than $16, \lambda^{1}=16$ and $\lambda^{2}=15$.

By $T(\alpha)$ we will denote the set of the pairs $\left\{(i j): \lambda_{i j}=\right.$ $\alpha$, e.g., $T\left(\lambda^{k}\right)$ is the set of pairs having $\lambda_{i j}=\lambda^{k}$. For rest of the text, we say that a pair $(i j)$ is tight whenever either $y_{i j}=B_{i j}$ or at least one of the endpoints, say $i$, satisfies $y_{i}=L_{i}$. Thus a pair is tight whenever its connection strength cannot be increased being constrained by either connection budget or node capacities.

Before we characterize the recursive structure of stable solutions in Theorem 4.1, we give some intuition behind it. Suppose we are given a stable solution $(\vec{f}, \beta)$ for intermediary price $\beta$ such that $\lambda^{2}<\beta<\lambda^{1}$. Now using this solution, let us construct a stable solution $(\vec{y}, \gamma)$ for a lower intermediary price $\gamma$ such that $\lambda^{3}<\gamma<\lambda^{2}$ as follows: Initially, let $\vec{y}=\overrightarrow{0}$. Now let us set $y_{i j}=f_{i j}$ for all the pairs in $T\left(\lambda^{1}\right)$. This ensures that all the pairs in $T\left(\lambda^{1}\right)$ satisfy the conditions for stability (by virtue of $(\vec{f}, \beta)$ being stable). Now let us iteratively increase $y_{i j}$ for the pairs in $T\left(\lambda^{2}\right)$ until all such pairs are tight. We claim that this makes $(\vec{y}, \gamma)$ a stable solution. To see this, observe that none of the pairs in $T\left(\lambda^{2}\right)$ can violate the first condition for stability. Now let us check for the second condition of stability: All the pairs in $T\left(\lambda^{2}\right)$ have $\lambda_{i j}=\lambda^{2}>\gamma$. Also, by making these pairs tight, we have ensured that for every pair $(i j)$ in $T\left(\lambda^{2}\right)$ has at least one endpoint, say $i$, satisfying $y_{i}=L_{i}$ (note that $i$ also satisfies $\lambda_{i j} \leq \tau_{\text {min }}^{i}(\vec{y})$ by construction).

Thus all the pairs with $\lambda_{i j} \in\left\{\lambda^{1}, \lambda^{2}\right\}$ meet both the conditions for stability, and having $y_{i j}=0$ for the pairs with $\lambda_{i j}<\gamma<\lambda^{2}$ ensures that no other pair violates stability conditions. This proves that $(\vec{y}, \gamma)$ is a stable solution. It turns out that if $\gamma=\lambda^{2}$ then we need not ensure the tightness of all the pairs in $T\left(\lambda^{2}\right)$ while constructing $\vec{y}$.

It can be shown that the above approach of constructing stable solutions can be applied recursively to construct stable solutions for any price $\gamma$ and vice versa every stable solution can be constructed in such a manner. This gives us the following theorem:

Theorem 4.1 (Characterization) $A$ solution $(\vec{y}, \gamma)$ where $\gamma \in\left(\lambda^{k+1}, \lambda^{k}\right]$ is a stable solution if and only if there exists a stable solution $(\vec{f}, \beta)$ with $\beta \in\left(\lambda^{k}, \lambda^{k-1}\right)$ such that

(a) If $\gamma=\lambda^{k}$ then the allocation vector $\vec{y}$ is obtained by increasing $f_{i j}$ for all the pairs in $T\left(\lambda^{k}\right)$ in any arbitrary manner without violating the budget and capacity constraints.

(b) If $\gamma \in\left(\lambda^{k+1}, \lambda^{k}\right)$ then then the allocation vector $\vec{y}$ is obtained by increasing $f_{i j}$ for all the pairs in $T\left(\lambda^{k}\right)$ until all such pairs are tight.

Proof. [Theorem 4.1, Only if part]: Suppose we are given a stable solution $(\vec{y}, \gamma)$ with $\gamma \in\left(\lambda^{k+1}, \lambda^{k}\right]$. Now we need to prove that stable solution $(\vec{f}, \beta)$ exists as described in the theorem statement. 
The definition of stability implies that if $\lambda_{i j} \leq \lambda^{k+1}$ then $y_{i j}>0$. Hence we need to concentrate only on the pairs with $\lambda_{i j} \geq \lambda_{k}$ in order to prove the theorem. Given this, we will prove the theorem in two steps. In the first step, we will prove that a solution $(\vec{f}, \beta)$ is a stable solution where $\beta \in\left(\lambda^{k}, \lambda^{k-1}\right)$ and $\vec{f}$ is $\vec{y}$ restricted to $S(\beta)$ (where such a restriction means $f_{i j}=y_{i j}$ for $(i j) \in S(\beta)$ and $f_{i j}=0$ otherwise). In the second step, we will prove that $\vec{y}$ can be obtained by augmenting $\vec{f}$ as described in the theorem statement.

Let us begin with the first step. Consider the solution $(\vec{f}, \beta)$ where $\beta \in\left(\lambda^{k}, \lambda^{k-1}\right)$ and $\vec{f}$ is $\vec{y}$ restricted to $S(\beta)$. As observed before, $y_{i j}=0$ for all the pairs with $\lambda_{i j} \leq \lambda^{k+1}$. Thus restricting $\vec{y}$ to $S(\beta)$ to obtain $\vec{f}$ is equivalent to setting $y_{i j}=0$ for the pairs with $\lambda_{i j}=\lambda^{k}$. Given this, the first condition for the stability of $(\vec{f}, \beta)$ is straightforward to observe using stability of $(\vec{y}, \gamma)$. Now suppose there exists a pair for which the second condition of stability did not hold. Such a pair must have $\lambda_{u v}>\beta>\gamma$ and $y_{u v}=f_{u v}<B_{u v}$. Now we break the analysis into four cases:

(a) $f_{u}<L_{u}$ and $f_{v}<L_{v}$ : Stability of $(\vec{y}, \gamma)$ implies that for at least one endpoint, say $u, y_{u}=L_{u}$ and $\lambda_{u v} \leq$ $\tau_{\text {min }}^{u}(\vec{y})$. This together with $\lambda_{u v}>\beta$ tells us that for each pair $(u w) \ni u$ such that $y_{u w}>0$, we must have $\beta<\lambda_{u v} \leq \tau_{\text {min }}^{u}(\vec{y}) \leq \lambda_{u w}$. Thus restricting $\vec{y}$ to $S(\beta)$ would have ensured that $f_{u w}=y_{u w}$ for all $(u w) \ni u$. Hence $y_{u}=f_{u}=L_{u}$ which contradicts the assumption of $f_{u}<L_{u}$ made for this case.

(b) $\lambda_{u v}>\tau_{\text {min }}^{u}(\vec{f})$ and $\lambda_{u v}>\tau_{\text {min }}^{v}(\vec{f})$ : It is easy to observe that this implies $\lambda_{u v}>\max \left\{\tau_{\text {min }}^{u}(\vec{y}), \tau_{\min }^{v}(\vec{y})\right\}$. This implies that $(u v)$ violates the second stability condition in $(\vec{y}, \gamma)$ as both endpoints violate at least one inequality contradicting that $(\vec{y}, \gamma)$ is a stable solution.

(c) $\lambda_{u v}>\tau_{\text {min }}^{u}(\vec{f})$ and $f_{v}<L_{v}$ (with $\lambda_{u v} \leq \tau_{\text {min }}^{v}(\vec{f})$ ): Note that we assume $\lambda_{u v} \leq \tau_{\text {min }}^{u}(\vec{f})$ as otherwise we can use the analysis from the previous case. Observe that $\lambda_{u v}>\tau_{\text {min }}^{u}(\vec{f}) \geq \tau_{\text {min }}^{u}(\vec{y})$. Suppose if we also have $y_{v}<L_{v}$ then $(\vec{y}, \gamma)$ could not have been a stable solution as pair $(u v)$ would violate the second stability condition in the solution $(\vec{y}, \gamma)$ - this is because both endpoints would violate at least one inequality (namely $y_{v}<L_{v}$ and $\lambda_{u v}>\tau_{\min }^{u}(\vec{y})$ ). Hence it must be true that $y_{v}=L_{v}$, giving us $f_{v}<y_{v}$. This together with the construction of $\vec{f}$ implies that there must exist a pair $(v x)$ with $\lambda_{v x}=\lambda^{k}$ with $y_{v x}>0$. This gives us $\lambda_{u v}>\tau_{\text {min }}^{v}(\vec{y})$. We already know for this case $\lambda_{u v}>\tau_{\text {min }}^{u}(\vec{f}) \geq \tau_{\text {min }}^{u}(\vec{y})$. Hence we get $\lambda_{u v}>\max \left\{\tau_{\text {min }}^{u}(\vec{y}), \tau_{\text {min }}^{v}(\vec{y})\right\}$. This implies that $(u v)$ violates the second stability condition in $(\vec{y}, \gamma)$ as both endpoints violate at least one inequality contradicting that $(\vec{y}, \gamma)$ is a stable solution.

(d) $f_{u}<L_{u}$ and $\lambda_{u v}>\tau_{\text {min }}^{v}(\vec{f})$ : This is same as the previous case after exchanging the roles of $u$ and $v$.

This proves that $(\vec{f}, \beta)$ where $\beta \in\left(\lambda^{k}, \lambda^{k-1}\right)$ is a stable solution. This also completes the proof for $\gamma=\lambda^{k}$ as $\vec{f}$ was obtained by restricting $\vec{y}$ to $S(\beta)$ which was discussed above to be equivalent to setting $y_{i j}=0$ for the pairs with $\lambda_{i j}=\lambda^{k}$. Now suppose $\gamma \in\left(\lambda^{k}, \lambda^{k+1}\right)$. For every pair with $\lambda_{i j}=\lambda^{k}>\gamma$ with $y_{i j}<B_{i j}$, the second condition for stability applied to $(\vec{y}, \gamma)$ tells us that for at least one of the endpoints, say $i$, it holds that $y_{i}=f_{i}+\left(y_{i}-f_{i}\right)=L_{i}$. In other words, $\vec{y}$ is obtained by incrementing $f_{i j}$ by $y_{i j}-f_{i j}$ for all pairs with $\lambda_{i j}=\lambda^{k}$ until all such pairs are tight.

Proof. [If part, Theorem 4.1]: We will focus only on part (b) of the theorem statement in this case, as proof of part (a) follows similar reasoning.

Suppose there exists a stable solution $(\vec{f}, \beta)$ with $\beta \in$ $\left(\lambda^{k}, \lambda^{k-1}\right)$. Now we need to prove that a solution $(\vec{y}, \gamma)$ constructed from this solution as described in part(b) of theorem statement is a stable solution (where $\gamma \in\left(\lambda^{k+1}, \lambda^{k}\right)$ ).

For each pair $(i j)$, observe from the construction of $\vec{y}$ that $x_{i j}>0$ implies $y_{i j} \geq \gamma$ where $\gamma \in\left(\lambda^{k+1}, \lambda^{k}\right)$. Hence all the pairs satisfy the first condition of stability in the solution $(\vec{y}, \gamma)$. Now let us examine whether the second condition of stability holds for $(\vec{y}, \gamma)$. Notice that stability of $(\vec{f}, \beta)$ implies that whenever $\lambda_{i j}<\lambda^{k}$ then $f_{i j}=0$. This together with the construction of $\mathbf{y}$ gives us that $\lambda_{i j}<\lambda^{k}$ implies $y_{i j}=0$. Thus in order to examine whether the second condition holds for $(\vec{y}, \gamma)$, we need to concentrate only on the pairs with $\lambda_{i j} \geq \lambda^{k}$. We break the analysis into two cases:

1. Pairs $(i j)$ with $y_{i j}<B_{i j}$ and $\lambda_{i j}>\lambda^{k}$ : Since we have assumed the solution $(\vec{f}, \beta)$ to be stable, it means that for such a pair at least for one endpoint, say $i$ satisfies $y_{i}=L_{i}$ and $\lambda_{i j} \leq \tau_{\min }^{i}(\vec{y})$. Since such an endpoint $i$ has no more spare capacity, constructing $\vec{y}$ from $\vec{f}$ maintains $y_{i w}=f_{i w}$ for all pairs $(i w) \ni i$. This implies that for the pair $(i j)$ we also have $y_{i}=L_{i}$ and $\lambda_{i j} \leq \tau_{\min }^{i}(\vec{y})$. Hence we have proven that all the pairs with $y_{i j}<B_{i j}$ and $\lambda_{i j}>\lambda^{k}>\gamma$ satisfy the second condition of stability.

2. Pairs $(i j)$ with $y_{i j}<B_{i j}$ and $\lambda_{i j}=\lambda^{k}$ : For suc a pair at least one endpoint, say $i$, satisfies $y_{i}=L_{i}$ by construction of $\vec{y}$.

If $f_{i}=L_{i}$ then we have $y_{i j}=f_{i j}$ since there is no spare capacity left at node $i$. This gives us $\lambda^{k}=\lambda_{i j} \leq$ $\tau_{\text {min }}^{i}(\vec{x})$ along with $y_{i}=L_{i}$ (i.e, the second condition of stability is satisfied).

On the other hand if $f_{i}<L_{i}$ then for some pair (iw) $\ni i$ with $\lambda_{i w}=\lambda^{k}$, it must be true that $y_{i j}>0$ (since we already have $y_{i}=L_{i}$ as discussed above). This again gives us $\lambda_{i j}=\lambda^{k}=\lambda_{i w} \leq \tau_{\text {min }}^{i}(\vec{y})$ along with $y_{i}=L_{i}$. Hence we have proven that every pair ( $i j)$ with $y_{i j}<B_{i j}$ and $\lambda_{i j}=\lambda^{k}$ satisfy the second condition of stability.

This completes the proof of the if part of Theorem 4.1.

Notice that $\left(\overrightarrow{0}, \lambda^{0}\right)$ is the unique stable solution for a price $\gamma=\lambda^{0}$ where $\lambda^{0}$ denotes any arbitrary value greater than $\lambda^{1}$. Starting with $\left(\overrightarrow{0}, \lambda^{0}\right)$, we can keep constructing stable 
solutions recursively for lower prices using Theorem 4.1, to give us the following:

Theorem 4.2 There exists a stable solution $(\vec{y}, \gamma)$ for every constant price $\gamma \geq 0$. Moreover, whenever all $\lambda_{i j}$ are distinct, there exists a unique stable solution for any constant price $\gamma$ in the open interval $\left(\lambda^{k+1}, \lambda^{k}\right)$ for all $k \geq 1$.

Proof. Let us first prove that there exists a stable solution for every constant price $\gamma$. If $\gamma \in\left[\lambda^{1}, \infty\right]$, then it is easy to verify that $(\mathbf{0}, \gamma)$ is a stable solution. Suppose that $\gamma \in$ $\left[\lambda^{k+1}, \lambda^{k}\right)$. Now we can apply Theorem 4.1 recursively to obtain a stable solution for such a $\gamma \in\left[\lambda^{k+1}, \lambda^{k}\right)$. After a careful thought, we conclude that Theorem 4.1 holds even when $\gamma \in\left[0, \min _{(i j)} \lambda_{i j}\right)$.

For the uniqueness part when all $\lambda_{i j}$ are distinct, let us begin by observing that solution $(\mathbf{0}, \gamma)$ is the unique stable solution for $\gamma>\lambda^{1}$. Beginning with this solution and using the recursive approach described in Theorem 4.1(b), we obtain a uniquely stable solution for every $\gamma \in\left(\lambda^{k+1}, \lambda^{k}\right)$, since there is a unique pair to be made tight at each step of the recursive construction.

Note that Theorem 4.2 guarantees the existence of a stable solution for any constant price $\gamma$ independent of any other parameters (with the node payments to the intermediary being of the form $\gamma \cdot y_{i}$ ). However, Theorem 4.2 does not guarantee the existence of a stable solution for any particular pricing scheme. For example, it could be that for a pricing scheme in which the price is a function $p(y)$ of total connection strength $y$ (which is the case for proportional pricing with $p(y)=c(y) / y$ and marginal cost pricing with $p(y)=c^{\prime}(y)$ ), the only stable solutions guaranteed by the above theorem may have the form $(\vec{y}, \gamma)$ with $\gamma \neq p(y)$. Fortunately, we can prove Theorem 4.3, which guarantees the existence of stable solutions for a large class of pricing schemes.

Theorem 4.3 (Existence) There exists a stable solution of the form $(\vec{y}, p(y))$ for any continuous non-decreasing price function $p(\cdot)$ with $p(0)=0$.

The proof of the above theorem involves beginning with the stable solution $\left(\overrightarrow{0}, \lambda^{0}\right)$ (where $\lambda^{0}$ is any value greater than $\left.\lambda^{1}\right)$, which has a total connection strength of 0 . Then we recursively construct solutions as described in Theorem 4.1, slowly increasing the total connection strength of the allocation vector in the process until the total connection strength and intermediary price satisfy the relation prescribed by the price function $p(\cdot)$. Now we describe the proof in details:

Proof. [Theorem 4.3] Recall that $\lambda^{0}$ denotes some arbitrary value greater than $\lambda^{1}$. Let $\lambda^{m}$ denote $\min _{i j} \lambda_{i j}$ and let $\lambda^{m+1}=0$. Let $\vec{f}_{0}$ denote the zero vector $\overrightarrow{0}$. Consider a sequence of solutions $\left\{\left(\vec{f}_{k}, \gamma^{k}\right)\right\}_{k=0}^{m}$ where $\gamma^{k} \in\left[\lambda^{k}, \lambda^{k+1}\right)$ and the allocation vector $\vec{f}_{i}$ is obtained recursively from $\vec{f}_{i-1}$ as specified Theorem 4.1(b) by making all the pairs in $T\left(\lambda^{k}\right)$ tight. Observe that all these solutions are tight for $\gamma^{k} \in\left[\lambda^{k}, \lambda^{k+1}\right)$ as any solution obtained in part(b) of Theorem 4.1 is also stable for intermediary price of $\lambda^{k}$ in Theorem 4.1 part (a). We break the further analysis into two cases: Case $p\left(f_{m}\right)<\lambda^{m}$ : As $\left(\overrightarrow{f_{m}}, \gamma^{m}\right)$ is a stable solution for $\gamma^{m} \in\left[\lambda^{m}, \lambda^{m+1}\right)$ where $\lambda^{m+1}=0$, we conclude that $\left(\overrightarrow{f_{m}}, p\left(f_{m}\right)\right)$ is a stable solution.

Case $p\left(f_{m}\right) \geq \lambda^{m}$ : This together with $p(0)=0$ tells us that there exists a $\lambda^{k}$ such that $p\left(f_{k-1}\right) \leq \lambda^{k-1}$ and $p\left(f_{k}\right) \geq \lambda^{k}$. Thus we know that there exists an $\alpha \in[0,1]$ such that $p\left(f_{k-1}+\alpha \cdot\left(f_{k}-f_{k-1}\right)\right)=\lambda^{k}$ using continuity of $p(\cdot)$. This gives us $p(\vec{g})=\lambda^{k}$ where $\vec{g}=\vec{f}_{k-1}+\alpha\left(\overrightarrow{f_{k}}-\overrightarrow{f_{k-1}}\right)$. Applying Theorem 4.1 (part (a)), we get that $\left(\vec{g}, \lambda^{k}\right)$ is a stable solution. This together with the already known fact $p(\vec{g})=\lambda^{k}$ completes the proof for this remaining case.

Since we assume $c(y)$ is strictly convex with $c^{\prime}(0)=c(0)=$ 0 , the marginal cost pricing scheme satisfies Theorem 4.3. It can also be shown that under these conditions $c(y) / y$ is increasing function of $y$. Thus setting $c(0) / 0=0$ makes Theorem 4.3 hold for proportional pricing.

\section{Price of Anarchy}

In this section, we provide our main results on bounding the worst-case efficiency (PoA) of the above pricing schemes. Hence having a small PoA (See Equation 5) implies that the quality of all stable solutions will be close to the social optimum despite of nodes behaving in a self-interested manner.

Indeed, we show that under marginal cost pricing PoA is upper bounded by 2 , and thus all stable networks of connections are close to optimum in cost. We also show that under proportional pricing PoA is upper bounded by $\max \left(2, \sup _{y \geq 0} \frac{y c^{\prime}(y)}{c(y)}\right)$. For example, under a common assumption that the operating $\operatorname{cost} c(y)$ of the intermediary is a convex polynomial of degree at most $d$, this bound becomes at most $d$, making proportional pricing very reasonable as long as the operating cost of the intermediary is not too convex.

The critical step for proving both these PoA results is to prove Lemma 5.3 which states that to bound PoA, we need to consider only those instances with all pairs having equal $\lambda_{i j}$ values. However, before that we will first state some elementary observations, Propositions 5.1-5.2 and Lemma 5.1-5.2 which will be used later to prove our main results.

Observation 5.1 If $a, b, c, d$ are positive numbers, with $b-$ $d>0$ and $a / b>c / d$ then $\frac{a-c}{b-d}>\frac{a}{b}$.

Observation 5.2 If $a, b, c, d, x, y$ are positive numbers such that $x>y$ and $a / b>c / d$ then $\frac{a+y c}{b+y d}>\frac{a+x c}{b+x d}$

The following propositions 5.1 and 5.2 allow us to perform minor perturbations in $\lambda_{i j}$ values of a set of pairs while maintaining stability of a solution $(\vec{y}, \gamma)$. Before we state them, recall that $T(\alpha)$ denotes the set of pairs $\left\{(i j): \lambda_{i j}=\alpha\right\}$.

Proposition 5.1 If $(\vec{y}, \gamma)$ is a stable solution with $\gamma \leq \lambda^{k}$, then it is also stable in the instance obtained by changing $\lambda_{i j}$ for every $(i j) \in T\left(\lambda^{k-1}\right)$ to some fixed $\Lambda \in\left[\lambda^{k}, \lambda^{k-2}\right)$.

Proof. We need to concentrate only on the pairs satisfying $\lambda_{i j}=\lambda^{k-1}$ as it can easily be observed that even after transforming the instance as specified in the claim, every other pair continues to satisfy both conditions for stability. It is immediate that all the pairs whose $\lambda_{i j}$ was perturbed satisfy the first condition of stability even after the transformation since $\gamma \leq \lambda^{k}$. Also, as $(\vec{y}, \gamma)$ was stable before the transformation 
with $\lambda^{k-1}>\lambda^{k} \geq \gamma$, all such pairs must have at least one endpoint (say $i$ ) such that $y_{i}=L_{i}$ and $\lambda^{k-1} \leq \tau_{\text {min }}^{i}(\vec{y})$. This does not change even after the transformation hence completing the proof.

The following proposition is immediate after noticing that if $(\vec{y}, \gamma)$ is a stable solution then every pair $(i j)$ with $\lambda_{i j}<\gamma$ has $y_{i j}=0$.

Proposition 5.2 If $(\vec{y}, \gamma)$ is a stable solution with $\gamma>\lambda^{k}$, then it is also stable in the instance obtained by changing $\lambda_{i j}$ for all $(i j) \in T\left(\lambda^{k}\right)$ to some fixed $\Lambda \in(0, \gamma)$.

Lemma 5.1 If $(\vec{y}, \gamma)$ is a stable solution with $\gamma<\lambda^{1}$ and $\vec{g}$ is any other allocation vector then

$$
\sum_{i} \sum_{\substack{(i j) \ni i \\ \text { s.t. }(i j) \in T\left(\lambda^{1}\right)}} B_{i j} \geq \sum_{i} \sum_{\substack{(i j) \ni i \\ \text { s.t. }(i j) \in T\left(\lambda^{1}\right)}}\left(2 \cdot g_{i j}-y_{i j}\right)
$$

Proof. Consider the allocation vector $\vec{z}$ obtained by setting $z_{i j}=y_{i j}$ for $(i j) \in T\left(\lambda^{1}\right)$ and $z_{i j}=0$ otherwise. In other words, $\vec{z}$ is obtained by restricting $\vec{y}$ to $T\left(\lambda^{1}\right)$. This construction makes all the pairs in $T\left(\lambda^{1}\right)$ satisfy both the conditions for stability with the allocation vector $\vec{z}$. Thus the solution $(\vec{z}, \gamma)$ becomes stable if we eliminate all the pairs with $\lambda_{i j}<\lambda^{1}$. Thus proving the above lemma is equivalent to proving the following claim:

Claim: For an instance $I$ with all pairs $(i j)$ having same value of $\lambda_{i j}=\lambda$, if $(\vec{z}, \gamma)$ is a stable solution with $\gamma<\lambda$ and $\vec{g}$ is any other allocation vector then

$$
\sum_{i} \sum_{(i j) \ni i} B_{i j} \geq 2 \cdot \sum_{i} \sum_{(i j) \ni i} g_{i j}-\sum_{i} \sum_{(i j) \ni i} z_{i j}
$$

Notice that it is sufficient to prove the above claim taking $\vec{g}$ to be the allocation vector with maximum value of $\sum_{i j} g_{i j}$ and we will assume $\vec{g}$ to be such a vector for rest of the proof. Let $\vec{f}$ denote the flow vector obtained by setting $f_{u v}=\min \left\{z_{u v}, g_{u v}\right\}$ from every pair $(u v)$ (i.e., $\vec{f}$ is the common component of $\mathbf{z}$ and $\mathbf{g}$ ). Consider another instance $I_{1}$ obtained from $I$ by iterating over all the pairs $(u v)$ and subtracting $f_{u v}$ from each $B_{u v}, L_{u}$ and $L_{v}$ in each iteration. Let $L_{u}^{\prime}$ denote the capacity of node $u$ in and $B_{u v}^{\prime}$ denote the budget corresponding to $(u v)$ in the instance $I_{1}$.

Notice that in the instance $I_{1}$, the objective of a node $C_{i}(\vec{y}, \gamma)$ (See Equation 1) is equivalent to maximizing $\sum_{(i j) \ni i} y_{i j}$ since all the pairs have equal $\lambda_{i j}$ 's and the intermediary price satisfies $\gamma<\lambda$. With these settings the stability conditions for $(\vec{y}, \gamma)$ reduce to a simple form that $y_{i j}<B_{i j}^{\prime}$ implies that least one of the endpoints, say $i$, satisfies $y_{i}=L_{i}^{\prime}$. This stability condition and the equivalent objective of maximizing $\sum_{(i j) \ni i} y_{i j}$ is the same as authors have in [Anshelevich et al., 2013] for M2H games with $\alpha=0$. By our construction, it can also be seen that $\vec{z}-\vec{f}$ is a stable solution in $I_{1}$. Although in $\mathrm{M} 2 \mathrm{H}$ games with $\alpha=0$ the authors have budgets $B_{i j} \in\{0,1\}$ and node capacities are assumed to be integral, we can adapt the proof of (Theorem 10 part 1 , keeping $\alpha=0$ ) in [Anshelevich et al., 2013] to obtain the following:

$$
2 \cdot \sum_{i} \sum_{(i j) \ni i}\left(z_{i j}-f_{i j}\right) \geq \sum_{i} \sum_{(i j) \ni i}\left(g_{i j}-f_{i j}\right)
$$

With simple algebraic manipulations, the above equation can also be written as

$$
\sum_{i} \sum_{(i j) \ni i}\left(z_{i j}-f_{i j}\right) \geq \sum_{i} \sum_{(i j) \ni i}\left(g_{i j}-z_{i j}\right)
$$

From the instance $I$, we also have

$$
\sum_{i} \sum_{(i j) \ni i} B_{i j} \geq \sum_{i} \sum_{(i j) \ni i}\left(g_{i j}+z_{i j}-f_{i j}\right)
$$

Using Equation 6 in the above equation, we get the desired result.

The following lemma says that budgets $B_{i j}$ have a simplified form in an instance that achieves the worst-case PoA.

Lemma 5.2 Consider an instance that achieves the worstcase PoA with intermediary price function $p(\cdot)$. Then we have $B_{i j}=\max \left\{f_{i j}, g_{i j}\right\}$ where $\vec{g}$ denotes the allocation vector corresponding to the minimum social cost and $(\vec{f}, p(f))$ is a stable solution of maximum social cost in this instance.

Proof. The ratio $S C(\vec{f}) / S C(\vec{g})$ is given by

$$
\frac{S C(\vec{f})}{S C(\vec{g})}=\frac{c(f)+\sum_{i} \sum_{(i j) \ni i} \lambda_{i j} \cdot\left(B_{i j}-f_{i j}\right)}{c(g)+\sum_{i} \sum_{(i j) \ni i} \lambda_{i j} \cdot\left(B_{i j}-g_{i j}\right)}
$$

If $B_{i j}>\max \left(f_{i j}, g_{i j}\right)$ for any pair $(i j)$, then we can obtain another instance by reducing $B_{i j}$ by a tiny positive $\epsilon$ without affecting the stability of $(\vec{f}, p(f))$. Using Observation 5.1, it can be shown that in this process the ratio $S C(\vec{f}) / S C(\vec{g})$ strictly increases. Thus the original instance could not have been an instance achieving the worst-case PoA.

The following critical lemma says that worst-case PoA is achieved in an instance where all pairs have equal $\lambda_{i j}$.

Lemma 5.3 Consider an instance that achieves the worstcase PoA with price function $p(\cdot)$ and suppose that $P o A>2$. Then for all pairs $(i j)$ we have $\lambda_{i j}=\lambda$ for some $\lambda>0$.

Proof. Let $\tau_{\vec{y}}$ denote $\min \left\{\lambda_{i j}: y_{i j}>0\right\}$ for a given allocation vector $\vec{y}$, i.e., $\tau_{\vec{y}}$ corresponds smallest $\lambda_{i j}$ that a pair with positive connection strength can have in $\vec{y}$. In the instance that achives the worst-case PoA, let $\vec{g}$ be an allocation vector corresponding to the minimum social cost and let $(\vec{f}, p(f))$ be a stable solution of maximum social cost. This means that the ratio $S C(\vec{f}) / S C(\vec{g})$ equals the worst-case PoA in the instance under consideration.

The proof of the above lemma involves proving a series of claims, each requiring a careful analysis. The first claim involves proving that for the instance under consideration that achieves the worst-case PoA, $\tau_{\vec{g}} \geq \tau_{\vec{f}}$. The second claim involves showing that $\lambda_{i j} \in\left\{\tau_{\vec{g}}, \tau_{\vec{f}}\right\}$ for every pair $(i j)$. Given this, the final claims involves proving $\tau_{\vec{g}}=\tau_{\vec{f}}$.

The theme of proving all these claims is to make use of Proposition 5.1-5.2 to create an instance by perturbing $\lambda_{i j}$ 
values for some intelligently chosen class of pairs, while maintaining the stability of the solution $(\vec{f}, p(f))$. Later carefully applying Lemma 5.1-5.2 we show that in the newly created instance, the ratio $S C(\vec{f}) / S C(\vec{g})$ strictly increases. This contradicts our initial assumption that the original instance achieves the worst-case PoA. Now let us proceed to prove each of these individual claims.

Claim: $\tau_{\vec{g}} \geq \tau_{\vec{f}}$.

Suppose we had $\tau_{\vec{f}}>\tau_{\vec{g}}$ on the contrary. We break the further analysis into two cases:

(a) Case when $p(f)>\tau_{\vec{g}}$ : Recall that connection cost incurred by a pair $(i j)$ for a connection strength of $y_{i j}$ is given by $\lambda_{i j}\left(B_{i j}-y_{i j}\right)$. Now ee claim $p(f)>\tau_{\vec{g}}$, implies that the pairs in the class $T\left(\tau_{\vec{g}}\right)$ incur strictly positive connection cost in total for the allocation vector $\vec{f}$ but incur zero connection cost for the allocation vector $\vec{g}$. Note: recall that $T\left(\tau_{\vec{g}}\right)$ is the set of all pairs with $\lambda_{i j}=\tau_{\vec{g}}$.

Note that $p(f)>\tau_{\vec{g}}$ implies that the intermediary charges a price higher than $\tau_{\vec{g}}$ in the solution $(\vec{f}, p(f))$. Thus by the first stability condition, $f_{i j}=0$ for $(i j) \in$ $T\left(\tau_{\vec{g}}\right)$. This, together with Lemma 5.2 implies $B_{i j}=$ $g_{i j}$ for $(i j) \in T\left(\tau_{\vec{g}}\right)$. Hence the pairs in $T\left(\tau_{\vec{g}}\right)$ incur zero cost for the allocation vector $\vec{g}$. At the same time, the definition of $\tau_{\vec{g}}$ implies that at least one pair with $\lambda_{i j}=\tau_{\vec{g}}$ has $g_{i j}>0$. Combining it with $f_{i j}=0$ and $B_{i j}=g_{i j}$ (as discussed above), we get that the pairs in $T\left(\tau_{\vec{g}}\right)$ incur strictly positive cost in total for the allocation vector $\vec{f}$.

Now let us obtain another instance by increasing $\lambda_{i j}$ for all the pairs in $T\left(\tau_{\vec{g}}\right)$ to any fixed $\Lambda$ in the open interval $\left(p(f), \tau_{\vec{g}}\right)$ while Proposition 5.2 ensures the stability $(\vec{f}, p(f))$. Notice that in this procedure, the connection cost of the pairs in the set $T\left(\tau_{\vec{g}}\right)$ strictly increases for the allocation vector $\vec{f}$. However as $B_{i j}=g_{i j}$, they still incur zero connection cost for the allocation vector $\vec{g}$ even in the transformed instance. Thus during this transformation $S C(\vec{g})$ does not change but $S C(\vec{f})$ strictly increases. Thus the ratio $S C(\vec{f}) / S C(\vec{g})$ becomes strictly greater in the transformed instance, contradicting the assumption that the original instance achieves the worstcase PoA.

(b) Case when $p(f) \leq \tau_{\vec{g}}$ (and $\lambda^{1} \geq \tau_{\vec{f}}>\tau_{\vec{g}}$ ): In this case, we claim that the following holds true

$$
\begin{aligned}
& \lambda^{1} \cdot \sum_{i} \quad \sum_{(i j) \ni i}\left(B_{i j}-f_{i j}\right) \\
& \frac{\text { s.t. }(i j) \in T\left(\lambda^{1}\right)}{\lambda^{1} \cdot \sum_{i} \sum_{\substack{(i j) \ni i \\
\text { s.t. }(i j) \in T\left(\lambda^{1}\right)}}\left(B_{i j}-g_{i j}\right)} \leq 2
\end{aligned}
$$

To see it, suppose that the following held true:

$$
\sum_{(i j) \in T\left(\lambda^{1}\right)} f_{i j} \geq \sum_{(i j) \in T\left(\lambda^{1}\right)} g_{i j}
$$

If the condition in Equation 10 holds true then it trivially implies the bound in Equation 9. Now suppose that the condition in 10 does not hold. By our assumptions, we have $p(f) \leq \tau_{\vec{g}}<\tau_{\vec{f}}<\lambda^{1}$. Thus the bound given in Lemma 5.1 holds true. It can be shown that the left hand side term in Equation 9 gets maximized when the bound in Lemma 5.1 is met with equality to give us a desired upper bound of 2 .

Now if it were true that $P o A>2$ then we can create another instance by reducing $\lambda^{1}$ by a tiny $\epsilon$ such that $0<\epsilon<\lambda^{1}-\lambda^{2}$. Note that $(\vec{f}, p(f))$ still stays a stable solution in the transformed instance by Proposition 5.1. However, reduction in $\lambda^{1}$ leads to an increase in the ratio $S C(\vec{f}) / S C(\vec{g})$ by Observation 5.2. This contradicts the assumption that the original instance achieves the worstcase PoA.

Thus assuming $\tau_{\vec{f}}>\tau_{\vec{g}}$ leads us to contradictions in both above cases. Hence it must be true that $\tau_{\vec{g}} \geq \tau_{\vec{f}}$.

Claim: $\lambda_{i j} \in\left\{\tau_{\vec{f}}, \tau_{\vec{g}}\right\}$ for any pair $(i j)$.

We already know that $\tau_{\vec{g}} \geq \tau_{\vec{f}}$. We also know by Lemma 5.2 that $B_{i j}=0$ whenever $\lambda_{i j}<\tau_{\vec{f}} \leq \tau_{\vec{g}}$, thus we can ignore all such pairs. We break the further analysis into two cases:

(a) Suppose there exists a pair with $\lambda_{i j}>\tau_{\vec{g}}$ : This implies $\lambda^{1}>\tau_{\vec{g}}$. Now we claim that the following holds true

$$
\frac{\lambda^{1} \cdot \sum_{i} \sum_{\substack{(i j) \ni i \\ \text { s.t. }(i j) \in T\left(\lambda^{1}\right)}}\left(B_{i j}-f_{i j}\right)}{\lambda^{1} \cdot \sum_{i} \sum_{\substack{(i j) \ni i \\ \text { s.t. }(i j) \in T\left(\lambda^{1}\right)}}\left(B_{i j}-g_{i j}\right)} \leq 2
$$

To prove our claim, suppose that the following holds:

$$
\sum_{(i j) \in T\left(\lambda^{1}\right)} f_{i j} \geq \sum_{(i j) \in T\left(\lambda^{1}\right)} g_{i j}
$$

If the condition in Equation 10 holds true then it trivially implies the bound in Equation 9. Now suppose that the condition in 10 does not hold. We already know that $p(f) \leq \tau_{\vec{f}}$ from the first stability condition and the definition of $\tau_{\vec{f}}$. Combining this with our assumptions, we have $p(f) \leq \tau_{\vec{f}} \leq \tau_{\vec{g}}<\lambda^{1}$, giving us $p(f)<\lambda^{1}$. Thus the bound given in Lemma 5.1 holds true. It can be shown that the left hand side term in Equation 9 gets maximized when the bound in Lemma 5.1 is met with equality to give us a desired upper bound of 2 .

Now if it were true that $P o A>2$ then we can create another instance by reducing $\lambda^{1}$ by a tiny $\epsilon$ such that $0<\epsilon<\lambda^{1}-\lambda^{2}$. Note that $(\vec{f}, p(f))$ still stays a stable solution in the transformed instance by Proposition 5.1 . However, reduction in $\lambda^{1}$ leads to an increase in the ratio $S C(\vec{f}) / S C(\vec{g})$ by Observation 5.2. This contradicts the assumption that the original instance achieves the worstcase PoA.

(b) Suppose there exists a pair with $\tau_{\vec{g}}>\lambda_{i j}>\tau_{\vec{f}}$ : Note that by definition of $\tau_{\vec{g}}$ that these pairs satisfy $g_{i j}=0$. 
Thus for these pairs, $B_{i j}=f_{i j}$. Since we only allow $B_{i j}>0$ in our problem settings, we have $f_{i j}>0$ for all these pairs. This implies that these pairs have zero connection cost in the allocation vector $\vec{f}$ (because of $B_{i j}=f_{i j}$ ) but strictly positive connection cost in the allocation vector $\vec{g}$ (because of $g_{i j}=0$ ).

Now Consider all the pairs $(u v)$ which satisfy the condition $\lambda_{u v}=\min \left\{\lambda_{i j}: \tau_{\vec{g}}>\lambda_{i j}>\tau_{\vec{f}}\right\}$. By applying Proposition 5.1, we can reduce $\lambda_{u v}$ of all such pairs to $\tau_{\vec{f}}$ without affecting the stability of $(\vec{f}, p(f))$. In this process, the connection cost of all these pairs stays zero in the allocation vector $\vec{f}$ but strictly decreases in the allocation vector $\vec{g}$. Hence in this process $S C(\vec{f})$ does not change but $S C(\vec{g})$ strictly decreases, increasing the ratio $S C(\vec{f}) / S C(\vec{g})$ leading to a contradiction with the assumption that the original instance achieves the worstcase PoA.

Claim: $\tau_{\vec{g}}=\tau_{\vec{f}}$.

We already proved above that in the instance achieving worst-case PoA we have $\lambda_{i j} \in\left\{\tau_{\vec{f}}, \tau_{\vec{g}}\right\}$ for every pair $(i j)$ and $\tau_{\vec{g}} \geq \tau_{\vec{f}}$. Now if $\tau_{\vec{g}}>\tau_{\vec{f}}$, then we can apply the analysis from case (b) of proof of the claim $\tau_{\vec{g}} \geq \tau_{\vec{f}}$ to prove that we can construct another instance by reducing $\lambda^{1}=\tau_{\vec{g}}$ by a tiny $\epsilon$ keeping $(\vec{f}, p(f))$ stable while the ratio $S C(\vec{f}) / S C(\vec{g})$ strictly increases in this transformation. This leads to the contradiction that the original instance could achieve the worst-case PoA hence proving our claim.

Proving the above claim completes the proof of Lemma 5.3 as discussed in the beginning the proof.

Theorem 5.1 (PoA of proportional pricing) With proportional pricing, $P O A \leq \max \left(2, \sup _{f \geq 0} \frac{f \cdot c^{\prime}(f)}{c(f)}\right)$ where we define $c^{\prime}(0) / c(0)=0$.

Proof. Assume PoA $>2$ as otherwise there is nothing to prove. Consider an instance achieving worst-case PoA with proportional pricing. Let $\vec{g}$ be an allocation vector corresponding to the minimum social cost and let $(\vec{f}, p(f))$ be a stable solution of maximum social cost in this instance. Since we have assumed PoA $>2$, this implies that Lemma 5.3 holds. This gives us $\lambda_{i j}$ is the same for all pairs $(i j)$ and furthermore $\lambda_{i j}=\tau_{\vec{g}}=\tau_{\vec{f}}$ holds for each pair. Thus we get the following equation (where we denote $\sum_{i} \sum_{(i j) \ni i} B_{i j}$ by $B)$ :

$$
\frac{S C(\vec{f})}{S C(\vec{g})}=\frac{c(f)+\tau_{\vec{g}} \cdot(B-f)}{c(g)+\tau_{\vec{g}} \cdot(B-g)}
$$

We break the further analysis into two cases:

(a) Suppose $g>f$ : This gives us $c(f) / c(g)<1$. This lets us apply Observation 5.1 to Equation 11 gives us

$$
\frac{S C(\vec{f})}{S C(\vec{g})}=\frac{c(f)+\tau_{\vec{g}} \cdot(B-f)}{c(g)+\tau_{\vec{g}} \cdot(B-g)} \leq \frac{\tau_{\vec{g}} \cdot(B-f)}{\tau_{\vec{g}} \cdot(B-g)}=\frac{B-f}{B-g}
$$

We now claim that whenever $g>f$, we have $\lambda^{1}>\gamma$ (where $\gamma=c(f) / f$ for proportional pricing). We know that for the instance under consideration, all $\lambda_{i j}$ values are equal. Furthermore, we have $\tau_{\vec{g}} \geq c^{\prime}(g)$ from the optimality of $\vec{g}$ and using $g>f$ with convexity of $c(\cdot)$ also gives us $c^{\prime}(g)>c^{\prime}(f)>c(f) / f$. This gives us $\lambda^{1}=\tau_{\vec{g}}>\gamma$. Since all the $\lambda_{i j}$ are equal for the instance under consideration, this gives us $\lambda^{1}=\tau_{\vec{f}}=\tau_{\vec{g}}>$ $c(f) / f=\gamma$. Having $\lambda^{1}>\gamma$ lets us apply Lemma 5.1 to give us $B \geq 2 g-f$. Then we can apply Observation 5.1 to subtract $(B-(2 g-f))$ from both numerator and denominator on the right hand side of Equation 12 to obtain $S C(\vec{f}) / S C(\vec{g}) \leq 2$. This contradicts our initial assumption that PoA $>2$.

(b) Suppose $f \geq g$ : This implies $(B-f) \leq(B-g)$. This lets us use Observation 5.1 to subtract $\bar{\tau}_{\vec{g}} \cdot(B-f)$ from the numerator and the denominator of the ratio in Equation 11 to give us

$$
\begin{aligned}
\frac{S C(\vec{f})}{S C(\vec{g})} & \leq \frac{c(f)}{c(g)+\tau_{\vec{g}} \cdot(f-g)} \\
& \leq \frac{c(g)+c^{\prime}(f) \cdot(f-g)}{c(g)+\tau_{\vec{f}} \cdot(f-g)} \quad \ldots \text { by convexity } \\
& \leq \frac{c^{\prime}(f)}{\tau_{\vec{f}}} \ldots \text { by Observation } 5.1
\end{aligned}
$$

From the stability conditions and the definition of proportional pricing, we get $\tau_{\vec{f}} \geq \gamma=c(f) / f$. Substituting it in the above expression completes the proof of the theorem.

In particular, for a common assumption of $c(y)$ being a polynomial of degree $d$ (without a constant term as we assume $p(0)=0$ ), Theorem 5.1 gives us the following:

Theorem 5.2 PoA $\leq d$ with proportional cost sharing whenever the operating cost of intermediary $c(y)$ is a polynomial of maximum degree $d$ without a constant term.

Theorem 5.3 (PoA of marginal cost pricing) For marginal cost pricing, $P o A \leq 2$.

The proof of Theorem 5.3 is almost similar to that of Theorem 5.1 except that we use $\tau_{\vec{f}} \geq \gamma=c^{\prime}(f)$ in the very last step. It is worth noting that the above bound is tight, i.e., there exist stable solutions under marginal pricing which are a factor of 2 away from optimum.

To see the tightness of PoA bound with marginal cost pricing, consider an instance with 4 nodes $p, q, u, v$. Set $B_{p q}=B_{q u}=B_{u v}=1$ and let $\lambda_{p q}=\lambda_{q u}=\lambda_{u v}=100$. Let $B_{i j}=0$ as well $\lambda_{i j}=0$ for all other pairs. Set $L_{p}=L_{q}=L_{u}=L_{v}=1$. Let the intermediary cost function be $\epsilon y^{2}$, where $\epsilon>0$ is an extremely tiny constant, thus the only cost nodes need to consider while forming a connection is the connection cost and node capacities. It can be verified that $\vec{g}$ is an optimal allocation vector where $g_{p q}=g_{u v}=1$ and $g_{i j}=0$ for all other pairs. The solution $\left(\vec{f}, c^{\prime}(f)\right)$ is a stable solution in these settings with $f_{q u}=1$ (and $f_{i j}=0$ for all other pairs), as no other pair can increase their connection strength constrained by node capacities (see the second stability condition). Thus we have $S C(\vec{f}) \approx 200$ and $S C(\vec{g}) \approx 100$. This proves that the PoA bound for marginal cost pricing is tight. 
We would like to mention in passing that equal pricing where each node makes a payment of $c(y) / n$ to the intermediary has an unbounded worst-case efficiency. This happens because the connection costs of pairs with high $\lambda_{i j}$ can get subsidized because of the fees paid by the pairs with less beneficial connections (i.e., small $\lambda_{i j} B_{i j}$ ). As a result, in stable solutions the pairs with high $\lambda_{i j}$ can end up setting connections of very high strength to increase the operating costs $c(y)$ in a disproportionate manner. This does not happen in proportional pricing or marginal cost pricing as the payment $p(y) \cdot y_{i}$ made by a node $i$ depends on total connection strength $y$ as well as the strength of the connections $y_{i}$ formed by a node.

For an example of arbitrarily high worst-case efficiency with equal pricing, consider an instance with a pair $(v w)$ with $\lambda_{v w}=2$ and $B_{v w}=m$ with $2 m^{2}+1$ other nodes with tiny $\lambda_{i j}$ as well as tiny $B_{i j}$ for all other pairs. Let the intermediary function be $c(y)=y^{2}$. Let the node capacity be infinite for all the nodes. It can be verified that in the optimal solution, only pair of nodes to have non-zero connection strength is $(v w)$ and in the optimal allocation vector $\vec{g}$ will have $g_{v w}=1$ and $g_{i j}=0$ for the rest. Thus the minimum social cost $S C(\vec{g})$ is approximately $(m-1) \cdot 4+4=4 m$. With equal pricing, the price charged by the intermediary in a solution $\vec{y}$ to each node is $c(y) /\left(2 m^{2}+1\right) \leq c\left(\sum_{i} \sum_{j} B_{i j}\right) /\left(2 m^{2}+1\right)$. Since the pairs other than $(v w)$ has negligible budgets, this upper bound is strictly less than 2 in any solution. This together with Equation 4 and having infinite node capacities in the instance under consideration implies that in any candidate stable solution the pair $(v w)$ has a connection strength of its full budget $B_{v w}$. It can be verified that a solution $\left(\vec{f}, c(f) /\left(m^{2}+1\right)\right)$ is a stable solution with equal pricing where $f_{v w}=B_{v w}$ and $f_{i j}=0$ for all other pairs. Thus $S C(\vec{f})$ evaluates to approximately $4 m^{2}$. Thus we get $S C(\vec{f}) / S C(\vec{g}) \approx m$. This bound can be made arbitrarily high and hence equal pricing has an unbounded worst-case PoA.

\section{Conclusion}

Although different kinds of network formation games have been extensively studied in the literature, the natural setting where nodes need to pay an intermediary in order to form connections has not been explored. Based on the classic network formation game introduced in [Jackson and Wolinsky, 1996], and inspired especially by IXP pricing, we formulated a model of network formation games with intermediaries. For this model, we showed how to efficiently determine stable prices and allocations (and the fact that such prices exist) for a natural class of pricing schemes. Moreover, we proved that all stable solutions are close to optimal for marginal cost pricing (factor of 2 away), and the same is true for proportional pricing as long as the intermediary costs are not too convex.

\section{References}

[Ager et al., 2012] Bernhard Ager, Nikolaos Chatzis, Anja Feldmann, Nadi Sarrar, Steve Uhlig, and Walter Willinger. Anatomy of a large european IXP. In Proceedings of the ACM SIGCOMM 2012 conference on Applications, tech- nologies, architectures, and protocols for computer communication, pages 163-174. ACM, 2012.

[AMS-IX, 2015] AMS-IX. Amsterdam internet exchange pricing. https://ams-ix.net/ services-pricing/pricing, 2015. Retrieved on February 8, 2015.

[Anshelevich and Hoefer, 2012] Elliot Anshelevich and Martin Hoefer. Contribution games in networks. Algorithmica, 63(1-2):51-90, 2012.

[Anshelevich et al., 2013] Elliot Anshelevich, Onkar Bhardwaj, and Michael Usher. Friend of my friend: Network formation with two-hop benefit. In Algorithmic Game Theory, volume 8146, pages 62-73. Springer, Berlin Heidelberg, 2013.

[Augustin et al., 2009] Brice Augustin, Balachander Krishnamurthy, and Walter Willinger. IXPs: mapped? In Proceedings of the 9th ACM SIGCOMM conference on Internet measurement conference, pages 336-349. ACM, 2009.

[Bala and Goyal, 2000] Venkatesh Bala and Sanjeev Goyal. A noncooperative model of network formation. Econometrica, 68(5):1181-1229, 2000.

[Calvó-Armengol and İlkılıç, 2009] Antoni CalvóArmengol and Rahmi İlkılıç. Pairwise-stability and nash equilibria in network formation. International Journal of Game Theory, 38(1):51-79, 2009.

[Cardona Restrepo and Stanojevic, 2012] Juan Camilo Cardona Restrepo and Rade Stanojevic. IXP traffic: a macroscopic view. In Proceedings of the 7th Latin American Networking Conference, pages 1-8. ACM, 2012.

[Corbo and Parkes, 2005] Jacomo Corbo and David Parkes. The price of selfish behavior in bilateral network formation. In Proceedings of the twenty-fourth annual ACM symposium on Principles of distributed computing, pages 99-107. ACM, 2005.

[DEC-IX, 2015] DEC-IX. German internet exchange pricing. https://www.de-cix.net/ products-services/pricing/, 2015. Retrieved on February 8, 2015.

[Derks et al., 2008] Jean Derks, Jeroen Kuipers, Martijn Tennekes, and Frank Thuijsman. Local dynamics in network formation. In Proc. Third World Congress of The Game Theory Society, 2008.

[Dutta et al., 2005] Bhaskar Dutta, Sayantan Ghosal, and Debraj Ray. Farsighted network formation. Journal of Economic Theory, 122(2):143-164, 2005.

[Epstein et al., 2007] Amir Epstein, Michal Feldman, and Yishay Mansour. Strong equilibrium in cost sharing connection games. In Proceedings of the 8th ACM conference on Electronic commerce, pages 84-92. ACM, 2007.

[EuroIX, 2015] EuroIX. European internet exchange association: Starting an IXP - infrastructure and equipment. https://wWw.euro-ix.net/ starting-an-ixp, 2015. Retrieved on February 8, 2015. 
[Fabrikant et al., 2003] Alex Fabrikant, Ankur Luthra, Elitza Maneva, Christos H Papadimitriou, and Scott Shenker. On a network creation game. In Proceedings of the twentysecond annual symposium on Principles of distributed computing, pages 347-351. ACM, 2003.

[Jackson and Wolinsky, 1996] Matthew O Jackson and Asher Wolinsky. A strategic model of social and economic networks. Journal of economic theory, 71(1):44-74, 1996.

[Jackson, 2005] Matthew O Jackson. A survey of network formation models: stability and efficiency. Group Formation in Economics: Networks, Clubs, and Coalitions, pages 11-49, 2005.

[Johari, 2007] Ramesh Johari. The price of anarchy and the design of scalable resource allocation mechanisms. Algorithmic Game Theory, pages 543-568, 2007.

[Koutsoupias and Papadimitriou, 1999] Elias Koutsoupias and Christos Papadimitriou. Worst-case equilibria. In STACS 99, pages 404-413. Springer, 1999.

[LINX, 2015] LINX. London internet exchange service fees. https://www.linx.net/service/ servicefees.html, 2015. Retrieved on February 8, 2015.

[Page and Resende, 2013] Frank Page and Joana Resende. Network formation games. http:

//kenticoqa.uwb.edu/getattachment/

business/about/research-series/

network-formation-games-uwb.pdf, 2013.

[Papadimitriou, 2001] Christos Papadimitriou. Algorithms, games, and the internet. In Proceedings of the thirty-third annual ACM symposium on Theory of computing, pages 749-753. ACM, 2001.

[Ryan and Gerson, 2012] Patrick S Ryan and Jason Gerson. A primer on internet exchange points for policymakers and non-engineers. http://ssrn. com/abstract $=$ 2128103 , August 2012.

[Tardos and Wexler, 2007] Eva Tardos and Tom Wexler. Network formation games and the potential function method. Algorithmic Game Theory, pages 487-516, 2007.

[Vazirani, 2007] Vijay V Vazirani. Combinatorial algorithms for market equilibria. Algorithmic Game Theory, pages 103-134, 2007.

[Watts, 2003] Alison Watts. A dynamic model of network formation. In Networks and Groups, pages 337-345. Springer, 2003. 